\title{
Classical and Quantum Scattering on a Cone*
}

\author{
S. Deser ${ }^{1, \star \star}$ and R. Jackiw ${ }^{2}$ \\ ${ }^{1}$ Department of Physics, Boston University, 590 Commonwealth Avenue, Boston, MA 02215, \\ USA \\ ${ }^{2}$ Center for Theoretical Physics, Laboratory for Nuclear Science, and Department of Physics, \\ Massachusetts Institute of Technology, Cambridge, MA 02139, USA
}

\begin{abstract}
The "topological" scattering of a quantized test particle in the locally flat conical geometry of a localized source in 2+1-dimensional gravity is analyzed. Wave functions and scattering amplitudes are obtained and compared with those recently found in a different approach by 't Hooft. The propagator [heat kernel] is also determined.
\end{abstract}

\section{Introduction}

Einstein theory in $2+1$ dimensions is an appealing model for investigating the effects of quantized gravity because the full curvature is locally determined by matter. Consequently, there are no gravitational degrees of freedom and no gravitational interaction among localized sources since space-time is flat between them; dynamics is topology. As a preliminary, we investigated the properties of the classical theory some time ago [1-3] following earlier work [4]. There is also a close relation to cosmic strings in four dimensions since the space-time of an infinite straight string is effectively three-dimensional [5].

Recently, 't Hooft [6] has begun consideration of the quantum theory in terms of the interaction among quantized particle sources [gravity itself is of course only "quantized" through the sources]. In particular, he has analyzed two-particle scattering by examining the relative motion, which he reduces to that of a particle moving on a cone. In the present work, we shall treat the closely related problem: scattering of a test particle in the field of a stationary point mass, which gives rise to a locally flat, conical metric $[1,3,4]$. Hence we also need to find solutions of the Schrödinger equation on a cone. To this end, we use a partial wave expansion, adapted to the fact that there is no potential; the kinetic term carries the information that our space has conical geometry.

\footnotetext{
* This work is supported in part by funds provided by NSF grants PHY 82-01094, 88-04561 and the U.S. Department of Energy (D.O.E.) under contract \# DE-AC02-76ER03069

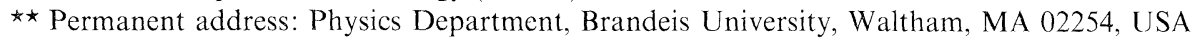


In Sect. II we describe and solve the classical scattering problem, then in Sect. III review the relevant aspects of the partial wave expansion for quantum scattering theory in the plane. The latter is applied in Sect. IV to the Schrödinger equation in conical space in order to obtain the phase shifts, wave functions and the scattering amplitude, whose unusual properties we discuss and in Sect. V compare with the results obtained in the quite different approach of ' $t$ Hooft [6]. In an Appendix we compute the propagator [heat kernel] for the Schrödinger equation on a cone.

\section{Conical Space and Classical Scattering}

The space-time interval in the presence at the origin of a stationary point particle with mass $M,(d s)^{2}=c^{2}(d t)^{2}-(d l)^{2}$, is characterized by the conical two-geometry of $(d l)^{2}$, which can be presented in various ways. In conformal coordinates, where an arbitrary number of stationary point particles is also easily described $[1,3,4]$,

$$
\begin{gathered}
(d l)^{2}=\frac{1}{R^{2(1-\alpha)}}\left[(d R)^{2}+R^{2}(d \Theta)^{2}\right], \quad 0 \leqq \Theta \leqq 2 \pi, \\
0 \leqq(1-\alpha) \equiv 4 G M<1 .
\end{gathered}
$$

Here $G$ is "Newton's constant." The range of $\alpha$ reflects the facts that space becomes a cylinder at $\alpha=0$ and that we are not interested in negative mass sources. For our purposes the conformal description is inconvenient owing to the awkward radial dependence. An intrinsic characterization uses a Euclidean metric with incomplete angular range $[1,3,4]$,

$$
(d l)^{2}=(d \varrho)^{2}+\varrho^{2}(d \varphi)^{2}, \quad-\pi \alpha \leqq \varphi \leqq \pi \alpha .
$$

This is the formulation employed by 't Hooft [6]. [He denotes his radial variable by $r$ instead of $\varrho$.$] The angular limitation leading to an excised region$ $-\pi \alpha>\varphi>\pi \alpha$ complicates the analysis. We shall work throughout with imbedded coordinates,

$$
(d l)^{2}=\alpha^{-2}(d r)^{2}+r^{2}(d \theta)^{2}, \quad 0 \leqq \theta \leqq 2 \pi,
$$

which describe a cone imbedded in flat three-space with the constraint $z=\sqrt{\left(\alpha^{-2}-1\right)\left(x^{2}+y^{2}\right)}$. This allows easy visualization of the classical motion and the complete angular range is appropriate for conventional partial wave analysis of the quantum regime. Translation among the above three coordinate frames is immediate through the relations

$$
r=R^{\alpha}=\alpha \varrho, \quad \theta=\Theta=\alpha^{-1} \varphi+\pi .
$$

The Lagrangian $L=\frac{m}{2}\left[\dot{x}^{2}+\dot{y}^{2}+\dot{z}^{2}\right]$ for a particle with mass $m$, constrained to move on the cone $z=\left(\alpha^{-2}-1\right)^{1 / 2} r$, becomes

$$
\begin{gathered}
L=\frac{m}{2} \dot{r}^{i} g_{i j} \dot{r}^{j}, \\
g_{i j} \equiv \delta_{i j}-\left(1-\alpha^{-2}\right) \hat{r}^{i} \hat{r}^{j},
\end{gathered}
$$


and gives the Hamiltonian

$$
\begin{gathered}
H=\frac{1}{2 m} p_{i} g^{i j} p_{j}, \\
g^{i j}=\delta_{i j}-\left(1-\alpha^{2}\right) \hat{r}^{i} \hat{r}^{\jmath} .
\end{gathered}
$$

The classical equations of motion

$$
\alpha^{-2} m \ddot{r}=m r \dot{\theta}^{2}, \quad \frac{d}{d t}\left(m r^{2} \dot{\theta}\right)=0
$$

have the usual first integrals,

$$
\begin{gathered}
\dot{\theta}=l / m r^{2}, \\
\frac{m \dot{r}^{2}}{2 \alpha^{2}}+\frac{l^{2}}{2 m r^{2}}=E,
\end{gathered}
$$

and are solved by

$$
\begin{aligned}
& r^{2}=\frac{2 E}{m} \alpha^{2}\left(t-t_{0}\right)^{2}+\frac{l^{2}}{2 m E}, \\
& \tan \alpha\left(\theta-\theta_{0}\right)=\frac{2 E \alpha}{l}\left(t-t_{0}\right) .
\end{aligned}
$$

The orbit equation reads

$$
r \cos \alpha\left(\theta-\theta_{0}\right)=\frac{l}{\sqrt{2 m E}} .
$$

[Note that when the above is expressed in intrinsic coordinates (2.3), $\alpha$ remains only in the combination of $l / \alpha$ : the motion is free on a flat plane, and the angular momentum is rescaled [1], a fact that will be especially relevant in the quantum case.]

Consider the scattering problem, i.e. the motion of the particle from $t=-\infty$ to $t=+\infty$. The scattering angle may be calculated by setting $\theta_{i}-\theta_{0}=-\pi / 2 \alpha$ as $t$ $\rightarrow-\infty$ [this defines the branch of $\tan ^{-1}$ on which the classical motion remains] and then noting that $\theta_{f}-\theta_{0}=+\pi / 2 \alpha$ as $t \rightarrow \infty$. The classical scattering angle $\omega$ is therefore

$$
\omega=\theta_{f}-\theta_{i}-\pi=\pi\left(\alpha^{-1}-1\right)
$$

This is the angle between the projections of the asymptotic motion onto the $x-y$ plane in the three space that embeds the cone. The scattering is backward $[\omega=(2 n+1) \pi]$ for even integer $\alpha^{-1}$, and there is no scattering $[\omega=2 n \pi]$ for $\alpha^{-1}$ odd, including of course flat space, $\alpha=1$. [In the intrinsic coordinates (2.3), $\varphi_{f}-\varphi_{i}=\pi$, so there is no conventional scattering. However, the trajectory "bends" owing to the identification of the edges of the excised region; consequently the undeflected direction subtends the angle $\pi \alpha$, which is to be subtracted from $\varphi_{f}-\varphi_{i}$, giving the $\varphi$-scattering angle $\alpha \omega$, in agreement with (2.14) and the transformation law (2.5).] 
Although we have described the dynamics non-relativistically, it should be clear that the relativistic motion is identical. One sees this most readily from the [relativistic] geodesic equation,

$$
\ddot{x}^{\mu}+\Gamma_{\alpha \beta}^{\mu} \dot{x}^{\alpha} \dot{x}^{\beta}=0,
$$

which coincides with the equation of motion (2.10). In (2.15), dots still denote differentiation with respect to ordinary time, because [ $g_{0 \mu}$ is Minkowskian] the proper time parameter can be chosen as time $(\vec{x}=0)$. Integration of the relativistic equations again produces (2.11)-(2.13), except that the constants of motion are renamed

$$
\left[l \rightarrow \frac{\operatorname{lm} c^{2}}{E}, E \rightarrow \frac{m c^{2}}{2}\left(1-\frac{m^{2} c^{4}}{E^{2}}\right)\right]
$$

\section{Quantum Scattering in the Plane}

Our scattering problem is not of the traditional potential type; the "interaction" modifies the kinetic term and is of the same derivative order. Nevertheless, we can formulate the quantum scattering problem associated with a planar Hamiltonian in a way that can be applied to a cone.

For any two-dimensional Hamiltonian $H$ with a complete set of solutions $\left\{u_{n}(r) e^{i n \theta}\right\}$,

$$
H u_{n}(r) e^{i n \theta}=E u_{n}(r) e^{i n \theta},
$$

such that

$$
u_{n}(r) \underset{r \rightarrow \infty}{\longrightarrow} \sqrt{\frac{2}{\pi \kappa(E) r}} \cos \left(\kappa(E) r-\frac{n \pi}{2}-\frac{\pi}{4}+\delta_{n}(E)\right),
$$

the superposition

$$
\psi(r, \theta)=\sum_{n} e^{i\left(\delta_{n}+n \pi / 2\right)} u_{n}(r) e^{i n \theta}
$$

behaves asymptotically as

$$
\psi(r, \theta) \underset{r \rightarrow \infty}{\longrightarrow} e^{i \kappa r \cos \theta}+\sqrt{\frac{i}{r}} f(\theta) e^{i \kappa r},
$$

where

$$
f(\theta)=\frac{1}{\sqrt{-2 \pi \kappa}} \sum_{n}\left(e^{2 i \delta_{n}}-1\right) e^{i n \theta} .
$$

This is easily checked by using the expansion

$$
e^{i z \cos \theta}=\sum_{n} i^{n} J_{n}(z) e^{i n \theta}
$$

together with the familiar asymptotic form of the Bessel functions $J_{n}$. The phase choice for $f(\theta)$ in (3.4) is for later convenience; it insures that

$$
\operatorname{Im} f(0)=\sqrt{\frac{\kappa}{8 \pi}} \int_{0}^{2 \pi} d \theta|f(\theta)|^{2}
$$


which expresses conservation of the probability current $\mathbf{j}, \nabla \cdot \mathbf{j}=0$, on the circle at infinity for wave functions with the asymptote (3.4), provided $f(\theta)$ is smooth on the entire circle.

Formulas (3.1)-(3.6) can be used on a cone when it is described by a complete angular range, as in our embedded coordinates. However, it will be seen that the resultant scattering amplitude is not smooth; consequently, probability conservation no longer implies (3.7).

When $\psi$ is decomposed into an incident wave $\psi_{\text {in }}$ and a scattered wave $\psi_{\text {sc }}$,

$$
\psi=\psi_{\mathrm{in}}+\psi_{\mathrm{sc}},
$$

one usually takes the respective asymptotes to be

$$
\begin{gathered}
\psi_{\mathrm{in}}(r, \theta) \underset{r \rightarrow \infty}{\longrightarrow} e^{i \kappa r \cos \theta}, \\
\psi_{\mathrm{sc}}(r, \theta) \underset{r \rightarrow \infty}{\longrightarrow} \sqrt{\frac{i}{r}} f(\theta) e^{i \kappa r} .
\end{gathered}
$$

We shall see that for the cone such a decomposition produces a scattering amplitude $f$ with delta function contributions, because the conical space is not asymptotically Euclidean and the test particle is never out of the "range of interaction." Therefore, it is useful to make an alternative decomposition in which the delta functions are removed from the scattering amplitude, at the price of changing $\psi_{\text {in }}$. This will be clarified presently.

\section{Quantum Scattering on a Cone}

The quantum Hamiltonian operator obtained from (2.8) with the replacement $\mathbf{p} \rightarrow \frac{\hbar}{i} \nabla$,

$$
H=-\frac{\hbar^{2}}{2 m} \partial_{i} g^{i j} \partial_{j}=-\frac{\hbar^{2}}{2 m}\left[\alpha^{2} \frac{1}{r} \partial_{r} r \partial_{r}+\frac{1}{r^{2}} \partial_{\theta}^{2}\right],
$$

is equivalent to the covariant formula

$$
H=-\frac{\hbar^{2}}{2 m} \frac{1}{\sqrt{g}} \partial_{i} \sqrt{g} g^{i j} \partial_{j},
$$

because $g \equiv \operatorname{det} g_{i j}=\alpha^{-2}=$ const. Therefore the radial wave function satisfies

$$
\left(r \partial_{r} r \partial_{r}-\alpha^{-2} n^{2}+\kappa^{2} r^{2}\right) u_{n}(r)=0
$$

where $\kappa^{2}=2 m E / \hbar^{2} \alpha^{2}$; note the dependence of $\kappa$ on $\alpha$. Of course $n$ is an integer, since $\theta$ has the full $2 \pi$ range.

[In the angular defect coordinates (2.3), the Schrödinger equation for $v_{l}(\varrho) e^{i l \varphi}$ implies $\left(\varrho \partial_{\varrho} \varrho \partial_{\varrho}-l^{2}+k^{2} \varrho^{2}\right) v_{l}(\varrho)=0, k^{2}=2 m E / \hbar^{2}$. The $l$ eigenvalue is determined by $e^{i l 2 \pi \alpha}=1$, i.e. $l=n / \alpha$ and the equation becomes as in (4.2) except $k=\alpha \kappa$. However, the accompanying change of radial variable $r=\alpha \varrho$, of (2.5), regains (4.2). In conformal coordinates (2.1), the equation following from (4.1b) for $U_{n}(R) e^{i n \Theta}, n$ 
integer, gives $\left(R \partial_{R} R \partial_{R}-n^{2}+R^{2 \alpha} \kappa^{2}\right) U_{n}(R)=0$, which reduces to (4.2) after the change of variables $(2.5), r=R^{\alpha}$. These equivalences are of course a manifestation of general covariance.]

We shall remain with the above non-relativistic formalism, but just as in the classical case, the relativistic quantum problem is no different. One should choose the Hamiltonian $\sqrt{m^{2} c^{4}+c^{2} p_{i} g^{i j} p_{j}}$, whose spectrum is found by diagonalizing the non-relativistic $H$ in (4.1); the relativistic energy eigenvalue is related to the eigenvalue in (4.2) by $E=\sqrt{m^{2} c^{4}+c^{2} \hbar^{2} \kappa^{2} \alpha^{2}}$.

We take the regular solution to (4.2),

$$
u_{n}(r)=(-1)^{\frac{n-|n|}{2}} J \frac{|n|}{\alpha}(\kappa r)
$$

whose asymptote is

$$
u_{n}(r) \underset{r \rightarrow \infty}{\longrightarrow} \sqrt{\frac{2}{\pi \kappa r}} \cos \left(\kappa r-\frac{|n| \pi}{2 \alpha}-\frac{\pi}{4}+\frac{(|n|-n) \pi}{2}\right) .
$$

Thus the phase shift is proportional to the classical scattering angle $\omega$ of (2.14),

$$
\delta_{n}=-\frac{|n| \pi}{2}\left(\alpha^{-1}-1\right)=-\frac{1}{2}|n| \omega,
$$

and the amplitude $f$ is

$$
f(\theta)=\frac{1}{\sqrt{-2 \pi \kappa}} \sum_{n}\left(e^{-i|n| \omega}-1\right) e^{i n \theta} .
$$

Regularization is needed to define the sums in (4.6), but it is clear by closure that

$$
S_{2}(\theta) \equiv \sum_{n} e^{i n \theta}=2 \pi \sum_{n} \delta(\theta-2 \pi n),
$$

since $\frac{1}{\sqrt{2 \pi}} e^{i n \theta}$ comprise the complete set of angular functions. As a check on our regularization procedure, which will also be used later in the construction of the complete wave function, we show how this comes about. Regularization is effected by inserting an exponential convergence factor into all sums,

$$
S_{2}(\theta)=\sum_{n=0}^{\infty}\left(e^{i n(\theta+i \varepsilon)}+e^{-i n(\theta-i \varepsilon)}\right)-1=\frac{1}{1-e^{i(\theta+i \varepsilon)}}+\frac{1}{1-e^{-i(\theta-i \varepsilon)}}-1 .
$$

As long as $\theta$ does not equal zero [or $2 \pi n]$ the " $i \varepsilon$ " may be dropped, and $S_{2}$ vanishes. For $\theta$ near 0 [or by periodicity, near $2 \pi n]$, the exponentials may be expanded leaving $\frac{1}{-i(\theta+i \varepsilon)}+\frac{1}{i(\theta-i \varepsilon)}=2 \pi \delta(\theta)$. Taking account of the periodic repetition gives (4.7). The remaining sum is treated similarly,

$$
\begin{gathered}
S_{1}(\theta) \equiv \sum_{n} e^{i(n \theta-|n| \omega)}=\sum_{n=0}^{\infty}\left(e^{i n(\theta-+i \varepsilon)}+e^{-i n(\theta+-i \varepsilon)}\right)-1, \\
\theta_{ \pm} \equiv \theta \pm \omega=\theta \pm \pi\left(\alpha^{-1}-1\right) .
\end{gathered}
$$


It is clear that the real part is like $S_{2}$,

$$
\frac{1}{2}\left(S_{1}(\theta)+S_{1}^{*}(\theta)\right)=\frac{1}{2}\left(S_{2}\left(\theta_{+}\right)+S_{2}\left(\theta_{-}\right)\right)=\pi \sum_{n}\left(\delta\left(\theta_{+}-2 \pi n\right)+\delta\left(\theta_{-}-2 \pi n\right)\right) .
$$

The imaginary part is easily evaluated to be

$$
\frac{1}{2 i}\left(S_{1}(\theta)-S_{1}^{*}(\theta)\right)=\frac{1}{2}\left[\operatorname{ctn} \frac{\theta_{-}}{2}-\operatorname{ctn} \frac{\theta_{+}}{2}\right],
$$

where the singularity is a principal value. Using trigonometric identities we may present $f$ as

$$
\sqrt{2 \pi \kappa} f(\theta)=\frac{\sin \omega}{\cos \omega-\cos \theta}-i \pi \sum_{n}(\delta(\theta+\omega-2 \pi n)+\delta(\theta-\omega-2 \pi n)-2 \delta(\theta-2 \pi n)) .
$$

Note that for generic $\omega$ both sides of (3.7) diverge [except for $\omega=2 \pi N$, where both sides vanish]. However, that equation is not a proper description of probability conservation in the present context, because $f$ is singular at $\theta= \pm \omega$ and periodic repetitions. Nevertheless, there is no doubt that probability is conserved: the wave functions satisfying the Schrödinger equation are nonsingular, hence the probability current is divergenceless.

As stated earlier, the separation of $\psi$ into $\psi_{\text {in }}$ and $\psi_{\mathrm{sc}}$ with asymptotes (3.9) is somewhat arbitrary. We can just as well consider that portion of $\psi_{\mathrm{sc}}$ that leads to the delta functions in $f(\theta)$ as belonging to the asymptote of the incoming wave. [Recall that the large-r asymptote of a plane wave is a radial wave times a "scattering amplitude" that is an angular delta function in the forward or backward direction.] Below, when we construct the complete wave function, we shall give this alternative separation for which the scattering amplitude has no delta functions and is the real portion of (4.12),

$$
\begin{gathered}
\psi=\tilde{\psi}_{\mathrm{in}}+\tilde{\psi}_{\mathrm{sc}}, \\
\tilde{\psi}_{\mathrm{sc}}(r, \theta)_{r \rightarrow \infty} \sqrt{\frac{i}{r}} \tilde{f}(\theta) e^{i \kappa r}, \\
\tilde{f}(\theta)=\frac{1}{\sqrt{2 \pi \kappa}} \frac{\sin \omega}{\cos \omega-\cos \theta} .
\end{gathered}
$$

Being real, $\tilde{f}$ cannot satisfy (3.7), even when that equation is modified to take into account the change in definition of the incoming wave. But as before, the remaining singularities of $\vec{f}-$ at $\pm \omega$ and periodic repetitions - invalidate (3.7), without contradicting probability conservation.

For even and odd integer $\alpha^{-1}, \omega=\pi N$ [classical backscattering and no scattering] $\tilde{f}$ vanishes - a curious "Ramsauer-Townsend"-like effect. For these values of $\alpha$, the scaled angular momentum $l=n / \alpha$ is an integer. Note that both the phase shifts and, apart from the kinematical $\kappa$-factor, the scattering amplitude are independent of the test particle mass, as expected from the equivalence principle. The phase shifts are also energy independent and increase with angular momentum as a consequence of the persistence of the "interaction" at large distance. 
Away from the special $\alpha$ values where the scattering amplitude vanishes, (4.13) reduces in the forward direction to

$$
f(0)=-\frac{1}{\sqrt{2 \pi \kappa}} \operatorname{ctn} \frac{\omega}{2},
$$

while in the backward direction we have

$$
\tilde{f}(\pi)=\frac{1}{\sqrt{2 \pi \kappa}} \tan \frac{\omega}{2} .
$$

For small source mass $4 G M \ll 1$ [but still large compared to test particle mass $M \gg m$ ], (4.13) reduces to [away from the forward and backward directions]

$$
f(\theta) \approx \sqrt{\frac{2 \pi}{\kappa} \frac{G M}{\sin ^{2} \theta / 2} .}
$$

Next we give a contour integral representation for the complete wave function $\psi(r, \theta)$ that solves the Schrödinger equation,

$$
\left(r \partial_{r} r \partial_{r}+\alpha^{-2} \partial_{\theta}^{2}+\kappa^{2} r^{2}\right) \psi(r, \theta)=0
$$

This representation will be used to separate $\psi$ into $\tilde{\psi}_{\text {in }}+\tilde{\psi}_{\text {sc }}$, in such a way that the scattering amplitude is $\tilde{f}$ of (4.13).

It is evident that any function of the form

$$
\psi(r, \theta)=\int_{C} d z e^{-i \kappa r \cos (z \pm \alpha \theta)} F(z)
$$

satisfies (4.15). The contour $C$ and weight function $F$ are determined by requiring $2 \pi$-periodicity in $\theta$ and by appropriate boundary conditions. We begin with $\psi(r, \theta)$ as specified by (3.3) and (4.5),

$$
\begin{aligned}
\psi(r, \theta) & =\sum_{n} a_{n} J \frac{|n|}{\alpha}(\kappa r) e^{i n \theta}, \\
a_{n} & =e^{-i \frac{|n|}{2}(\omega-\pi)} .
\end{aligned}
$$

From the Schläfli representation

$$
J_{v}(x)=\frac{1}{2 \pi} \int d z e^{-i x \sin z+i v z},
$$

where the contour runs from $-\pi+i \infty$, meeting the real axis at $-\pi$, passing along that axis to $\pi$, and then returning to $\pi+i \infty$, we have

$$
\begin{gathered}
\psi(r, \theta)=\frac{1}{2 \pi} \int d z e^{-i \kappa r \sin z} F(z, \theta), \\
F(z, \theta)=\sum_{n} e^{-i|n|\left(\frac{\omega}{2}-\frac{\pi}{2}-\frac{z}{\alpha}\right)} e^{i n \theta} \\
=\sum_{n=0}^{\infty}\left(e^{-i n\left(\frac{\omega}{2}-\frac{\pi}{2}-\frac{z}{\alpha}-\theta-i \varepsilon\right)}+e^{-i n\left(\frac{\omega}{2}-\frac{\pi}{2}-\frac{z}{\alpha}+\theta-i \varepsilon\right)}\right)-1 \\
=\frac{1}{2 i} \tan \frac{1}{2}\left(\frac{z}{\alpha}-\frac{\pi}{2 \alpha}+i \varepsilon+\theta\right)+\frac{1}{2 i} \tan \frac{1}{2}\left(\frac{z}{\alpha}-\frac{\pi}{2 \alpha}+i \varepsilon-\theta\right),
\end{gathered}
$$

with the necessary regularization inserted. 


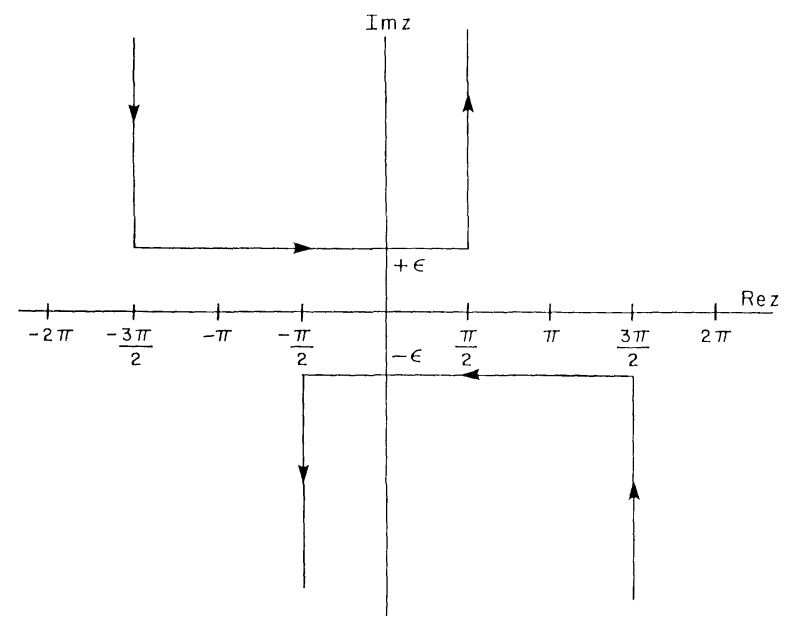

b

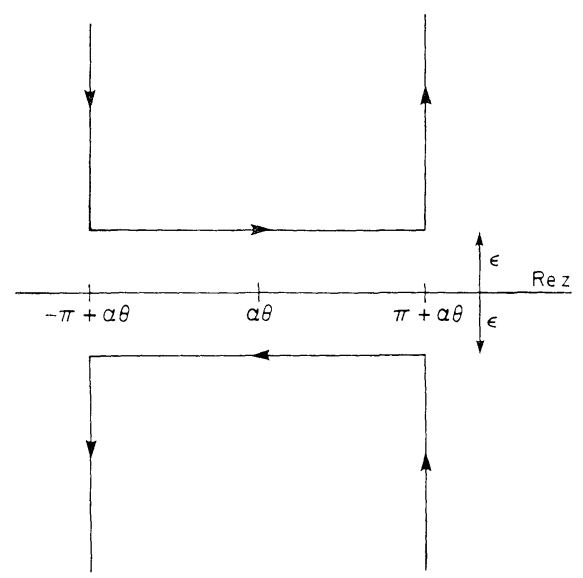

Fig. 1. a Contour of integration in (4.21a). b Contour of integration in (4.21b)

Upon substituting the two terms in the summed expression (4.20) into the integral (4.19), changing variables of integration, $z \rightarrow z+\frac{\pi}{2}-i \varepsilon$, and reflecting the variable of integration in the second term about the real and imaginary axes $(z \rightarrow-z)$ we arrive at

$$
\psi(r, \theta)=\frac{1}{4 \pi i} \int_{C_{1} \cup C_{2}} d z e^{-i \kappa r \cos z} \tan \frac{1}{2}\left(\frac{z}{\alpha}+\theta\right)=\psi(r, \theta+2 \pi),
$$

where the contours are depicted in Fig. 1a. These contours may be translated. It is easy to check that a shift by $\frac{\pi}{2}$ to the right for the upper contour and to the left for the lower contour is permitted. A final change of variables $z \rightarrow z-\alpha \theta$ yields the explicit determination of (4.16),

$$
\psi(r, \theta)=\frac{1}{4 \pi i} \int_{C} d z e^{-i \kappa r \cos (z-\alpha \theta)} \tan \frac{z}{2 \alpha},
$$

with $C$ given in Fig. $1 \mathrm{~b}$. 
We now separate $\psi(r, \theta)$ into two parts, which we shall show correspond to incident and scattered waves, the latter leading to $\tilde{f}$. This division is effected by reexpressing the contour in Fig. $1 \mathrm{~b}$ as the sum of vertical lines from $\pm i \infty$ to $\mp i \infty$, and a closed [Cauchy] contour around the real poles of $\tan \frac{z}{2 \alpha}$. The vertical contributions in Fig. 1 b give

$$
\tilde{\psi}_{\mathrm{sc}}(r, \theta)=\chi\left(r, \frac{\theta}{2}+\frac{\pi}{2 \alpha}\right)-\chi\left(r, \frac{\theta}{2}-\frac{\pi}{2 \alpha}\right)
$$

where

$$
\chi(r, \xi)=\frac{1}{4 \pi} \int_{-\infty}^{\infty} d y e^{i \kappa r \cosh y} \tan \left(\frac{i y}{2 \alpha}+\xi\right) .
$$

For large $r$,

$$
\chi(r, \xi) \underset{r \rightarrow \infty}{\longrightarrow} \frac{1}{4 \pi} e^{i \kappa r} \tan \xi \int_{-\infty}^{\infty} d y e^{i \frac{\kappa r}{2} y^{2}}=\sqrt{\frac{i}{8 \pi \kappa r}} e^{i \kappa r} \tan \xi .
$$

Equations (4.22)-(4.23) imply that

$$
\begin{aligned}
\tilde{\psi}_{\mathrm{sc}}(r, \theta) \underset{r \rightarrow \infty}{\longrightarrow} \sqrt{\frac{i}{r}} \frac{1}{\sqrt{8 \pi \kappa}}\left[\tan \left(\frac{\theta}{2}+\frac{\pi}{2 \alpha}\right)-\tan \left(\frac{\theta}{2}-\frac{\pi}{2 \alpha}\right)\right] e^{i \kappa r} \\
=\sqrt{\frac{i}{r}} \frac{1}{\sqrt{2 \pi \kappa}} \frac{\sin \omega}{\cos \omega-\cos \theta} e^{i \kappa r},
\end{aligned}
$$

i.e. $\tilde{\psi}_{\mathrm{sc}}$ is indeed a scattered wave whose scattering amplitude agrees with (4.13).

The contribution of the Cauchy contour determines $\psi-\tilde{\psi}_{\mathrm{sc}}$, i.e. $\tilde{\psi}_{\mathrm{in}}$, in terms of the [negative] residues of the poles of $\tan \frac{z}{2 \alpha}$ at $z=(2 n+1) \pi \alpha$ in the interval $(-\pi+\alpha \theta, \pi+\alpha \theta)$ :

$$
\tilde{\psi}_{\text {in }}(r, \theta)=\alpha \sum_{n}^{\prime} e^{-i \kappa r \cos \alpha \theta_{n}},
$$

where $\theta_{n} \equiv \theta-(2 n+1) \pi$, and the prime on the sum restricts the $n$ summation to $\alpha\left|\theta_{n}\right|<\pi$.

When $\theta= \pm \omega$, the scattering amplitude $\tilde{f}$ appears to diverge. However, at these points the contour representation for $\tilde{\psi}$ must be re-examined. One finds that the integral along one of the vertical lines is potentially infinite, but in fact vanishes by symmetry, since it is configured as a principal value: $[-\varepsilon, \varepsilon]$ is excluded. $\tilde{\psi}_{\mathrm{sc}}$ retains only the finite contribution from the remaining vertical contour, i.e. only the non-diverging tangent in (4.24) survives. Moreover, the pole that is now present at the edge of the interval, $\alpha\left|\theta_{n}\right|=\pi$, contributes only half its residue to $\tilde{\psi}_{\text {in }}$. At $\theta=\omega$ for example, the angular dependence in (4.24) reduces to the finite value $-\cot \omega$ [whose apparent divergence at $\alpha=\frac{1}{N}$ is spurious, since the full $\tilde{\psi}_{\text {sc }}$ then vanishes, see below]. We stress, however, that while $\tilde{f}(\theta= \pm \omega)$ is finite, $\tilde{f}$ is still singular since it grows arbitrarily large near those two angles. 
It is clear that the delta functions of (4.11a) that arise in our partial wave summation correspond to the poles from which $\tilde{\psi}_{\text {in }}$ is constructed. Including them in the incident wave evidently produces the result (4.25), which differs dramatically from the single $e^{i \kappa r \cos \theta}$ of (3.4). [Note that in the intrinsic coordinate $\varphi=\alpha(\theta-\pi)$, (4.25) is a superposition of plane waves propagating in various (rotated) directions.]

The incident wave depends on the "interaction" $\alpha-1$ and in the absence of the source, reduces to $e^{i \kappa r \cos \theta}$. Likewise by (4.22), $\tilde{\psi}_{\mathrm{sc}}$ vanishes at $\alpha=1$. Note that for generic $\alpha, \tilde{\psi}_{\text {in }}$ is discontinuous, but separately satisfies the Schrödinger equation (4.15) away from the discontinuities. The complete wave function (4.21) is manifestly continuous, so $\tilde{\psi}_{\mathrm{sc}}$ is needed to cancel the discontinuities of $\tilde{\psi}_{\mathrm{in}}$.

For $\alpha=\frac{1}{N}$ the discontinuities of $\tilde{\psi}_{\text {in }}$ disappear, $\tilde{\psi}_{\text {sc }}$ and $\tilde{f}$ vanish, leaving

$$
\psi(r, \theta)=\tilde{\psi}_{\text {in }}(r, \theta)=\alpha \sum_{n=[-1 / 2 \alpha]+1}^{[1 / 2 \alpha]} e^{-i \kappa r \cos \alpha \theta_{n}} .
$$

Here $[x]$ is the largest integer less than or equal to $x$. For example, when $\alpha=\frac{1}{2}$,

$$
\psi(r, \theta)=\tilde{\psi}_{\text {in }}(r, \theta)=\cos \kappa r \sin \frac{\theta}{2} \text {. }
$$
The "incident wave" is a standing wave-this is also true for all $\alpha=\frac{1}{2 N}$, where the
classical scattering is backward. For $\alpha=1 / 3$,

$$
\psi(r, \theta)=\tilde{\psi}_{\text {in }}(r, \theta)=\frac{1}{3}\left(e^{-i \kappa r \cos \left(\frac{\theta}{3}+\frac{\pi}{3}\right)}+e^{-i \kappa r \cos \left(\frac{\theta}{3}-\frac{\pi}{3}\right)}+e^{-i \kappa r \cos \left(\frac{\theta}{3}+\pi\right)}\right)
$$

$\tilde{\psi}_{\text {in }}$ is complex for all $\alpha=\frac{1}{2 N+1}$, where there is no classical scattering. It is easy to verify that the same formulas (4.27) can be derived from (4.17).

For rational $\alpha=\frac{p}{q}$, [integers $q>p>1$, with no common divisors] one may also construct a finite superposition of plane waves that solves $(4.15)$, and is $2 \pi$ periodic,

$$
\begin{aligned}
\psi_{0}(r, \theta) & =\frac{1}{q} \sum_{n=0}^{q-1} e^{-i \kappa r \cos \frac{p}{q} \theta_{n}} \\
= & \psi(r, \theta)+\psi\left(r, \theta+2 \pi\left(\frac{q}{p} \bmod 1\right)\right)+\ldots+\psi\left(r, \theta+2 \pi\left([p-1] \frac{q}{p} \bmod 1\right)\right) .
\end{aligned}
$$

[For irrational $\alpha$, no corresponding sum can be both finite and $2 \pi$-periodic, and this construction is not possible.] While $\psi_{0}$ is similar to $\tilde{\psi}_{\text {in }}$, it differs from (4.25) by being continuous, and the arguments of its exponentiated cosines are unrestricted. Since (4.28) is a superposition of plane waves, that expression shows no scattering. Of course (4.28) may also be presented as a sum of partial waves, as in (4.17a), except that the expansion coefficients $a_{n}$ no longer have the "scattering" form (4.17b): with the help of (3.6) one finds that those with $n$ an integer multiple of $p$ are unaltered, while the remaining ones vanish. 
For example for $\alpha=\frac{2}{3}$, the discontinuous $\tilde{\psi}_{\text {in }}$ from (4.25) is

$$
\tilde{\psi}_{\mathrm{in}}(r, \theta)= \begin{cases}\frac{2}{3}\left(e^{-i \kappa r \cos \left(\frac{2}{3} \theta-\frac{2 \pi}{3}\right)}+e^{-i \kappa r \cos \left(\frac{2}{3} \theta+\frac{2 \pi}{3}\right)}\right) & 0 \leqq \theta<\frac{\pi}{2} \\ \frac{2}{3} e^{-i \kappa r \cos \left(\frac{2}{3} \theta-\frac{2 \pi}{3}\right)} & \frac{\pi}{2}<\theta<\frac{3 \pi}{2} \\ \frac{2}{3}\left(e^{-i \kappa r \cos \left(\frac{2}{3} \theta-\frac{2 \pi}{3}\right)}+e^{-i \kappa r \cos \frac{2}{3} \theta}\right) & \frac{3 \pi}{2}<\theta \leqq 2 \pi .\end{cases}
$$

The corresponding scattering amplitude is $-(2 \pi \kappa)^{-1 / 2} \sec \theta$. The plane wave superposition that is periodic, continuous [all three components of (4.29) being present in the entire $[0,2 \pi]$ interval], satisfies the Schrödinger equation with $\alpha=\frac{2}{3}$ and does not scatter is constructed from (4.28) as

$$
\psi_{0}(r, \theta)=\frac{1}{3}\left(e^{-i \kappa r \cos \left(\frac{2}{3} \theta-\frac{2 \pi}{3}\right)}+e^{-i \kappa r \cos \left(\frac{2}{3} \theta+\frac{2 \pi}{3}\right)}+e^{-i \kappa r \cos \frac{2}{3} \theta}\right)=\psi_{0}(r, \theta+\pi) \text {. }
$$

The partial wave expansion of (4.30) is as in (4.17), with only even partial waves contributing, which means that

$$
\psi_{0}(r, \theta)=\frac{1}{2}(\psi(r, \theta)+\psi(r, \theta \pm \pi)) .
$$

Equation (4.31) may also be understood in the following manner. If we free our wave functions from the convention that the $\boldsymbol{\kappa}$ is oriented along the $x$-axis, then a wave function for arbitrary $\mathbf{\kappa}=\left(\kappa, \theta_{\kappa}\right)$ is obtained from the conventional one by replacing $\theta$ by $\theta-\theta_{\kappa}$. Thus, (4.31) shows that $\psi_{0}$ is a superposition of two scattering solutions, one with $\boldsymbol{\kappa}$, the second with $-\boldsymbol{\kappa}$. The generic case with $\alpha=\frac{p}{q}$ is similar. We also note that more general, non-scattering wave functions may be constructed: e.g., $\psi_{0}(r, \theta)+\psi_{0}(r, \theta+\gamma)$ for any $\gamma$.

\section{Discussion}

We have described the conical quantum scattering problem in two complementary ways. In the first, (3.8) and (3.9), appropriate to a partial wave expansion, the incoming wave is the conventional plane wave, but the scattering amplitude of (4.12) not only is singular near $( \pm)$ the classical scattering angle $\omega$ [and its periodic repetitions], but also contains delta functions. In the second, (4.13), (4.24), (4.25), it is recognized that the delta functions may be moved to the incident wave, which becomes a discontinuous superposition of plane waves, with an associated real scattering amplitude $\tilde{f}$ in (4.13), equal to $f$ except for the delta functions. Both approaches provide a decomposition of the same non-singular solution to the Schrödinger equation. Consequently, unitarity is never in jeopardy, although no conventional optical theorem is available in either formulation.

We have also obtained, for rational $\alpha$, a set of simple solutions (4.28) that do not scatter; the total wave function here is a continuous version of the corresponding $\tilde{\psi}_{\text {in }}$ and is constructed by taking a basic incoming plane wave and completing it 
with its periodic recurrences, a procedure which terminates if $\alpha$ is rational. This solution is also given by linear combinations of scattering solutions, with variously directed propagation vectors, but it differs from the scattering solution in that it really corresponds to a "tuned" set of separate complete plane waves, unlike those in $\tilde{\psi}_{\text {in }}$, which are not all present in the full $[0,2 \pi]$ angular range. In the Appendix, this difference will appear as an unorthodox propagator $G_{0}$ whose zero time limit is a sum of several spatial delta functions, rather than just one.

't Hooft's analysis [6] of the 2+1-dimensional scattering problem uses the intrinsic conical coordinates throughout, avoiding a partial wave decomposition. It is easy to compare his formulas with ours. Denoting our results at energy $E=\frac{\hbar^{2} \kappa^{2} \alpha^{2}}{2 m}$ with the subscript $\kappa$, the corresponding intrinsically conical ex$2 m$
pressions [superscript $c$ ] at energy $E=\frac{\hbar^{2} k^{2}}{2 m}$ with the subscript $k$, and recalling the transformation law (2.5), allows establishing the following connection formula for the scalar $\psi$ :

$$
\psi_{k}^{c}(\varrho, \varphi)=\psi_{k=k / \alpha}\left(r=\varrho \alpha, \theta=\alpha^{-1} \varphi+\pi\right) .
$$

Transformation of our expressions (4.13), (4.21b), (4.22), and (4.25) into conical coordinates according to (5.1) shows that they agree with ' $t$ Hooft's, except for an overall normalization factor $\alpha$ that multiplies our formulas relative to his - our wave function is normalized to unity at the origin. [The contours in our formula for the complete wave function ( $4.21 \mathrm{~b}$ ), are configured in the same way as ' $t$ Hooft's, but are displaced relative to his. Since he does not label his figure explicitly, we do not know whether the difference is significant. It must not be, because the separate parts of the wave functions do agree.]

't Hooft argues that the calculation also describes two-body scattering in the center of mass system. While our investigation does not address the issue of reducing the two-body problem to an effective one-body problem, it is clear that for heavy masses and slow relative motion this is true. Because it is known that the space-time of two static masses is a spatial cone with deficit angle to the summed masses plus a further excision whose relative importance diminishes with increasing masses [1], it is reasonable that to leading order the conical space-time describes slowly moving, heavy particles.

\section{Appendix}

We compute the propagator

$$
G\left(\mathbf{r}, \mathbf{r}^{\prime} ; t\right)=\left\langle\mathbf{r}^{\prime}\left|e^{-i t H}\right| \mathbf{r}\right\rangle
$$

for the conical Schrödinger equation; i.e. we obtain a solution to the Schrödinger Eq. (4.15) that reduces to a spatial delta function at initial time. We work with imaginary time $T=i t$; (A.1) then is the heat kernel.

With the complete set of energy eigenstates, $J_{|n| \mid \alpha}(\kappa r) e^{i n \theta}$ labeled by $\kappa$ and $n$, the following representation may be given for $G$ :

$$
\begin{gathered}
G\left(\mathbf{r}, \mathbf{r}^{\prime},-i T\right)=\frac{1}{2 \pi} \int_{0}^{\infty} \kappa d \kappa e^{-\frac{\tau \kappa^{2}}{2}} \sum_{n} J_{|n| / \alpha}\left(\kappa r^{\prime}\right) J_{|n| / \alpha}(\kappa r) e^{i n \Delta \theta}, \\
\Delta \theta \equiv \theta^{\prime}-\theta, \quad \tau=T \frac{\hbar^{2} \alpha^{2}}{m} .
\end{gathered}
$$


The integration, whose measure is determined by the normalization of our wave functions, yields a modified Bessel function.

$$
G\left(\mathbf{r}, \mathbf{r}^{\prime} ;-i T\right)=\frac{1}{2 \pi \tau} e^{-\frac{r^{2}+r^{\prime 2}}{2 \tau}} \sum_{n} e^{i n \Delta \theta} I_{|n| / x}\left(\frac{r r^{\prime}}{\tau}\right) .
$$

The summation is performed with the help of the Schläfli representation for $I_{v}$,

$$
I_{v}(x)=\frac{1}{2 \pi} \int_{C} d x e^{x \cos z+i v z},
$$

where the contour is the same as in (4.18). Use of this in (A.3) allows performing the $n$ sum, after it has been regulated through multiplication by $e^{-\varepsilon|n|}$. In a now familiar way, one arrives at the difference of half-angle cotangents, evaluated at $\Delta \theta \pm\left(\frac{z}{\alpha}+i \varepsilon\right)$. Further shifts of integration variables gives

$$
G\left(\mathbf{r}, \mathbf{r}^{\prime} ;-i T\right)=\frac{i}{8 \pi^{2} \tau} \int_{C_{1} \cup C_{2}} d z\left(\operatorname{ctn} \frac{z}{2 \alpha}\right) \exp -\frac{1}{2 \tau}\left(r^{2}+r^{\prime 2}-2 r r^{\prime} \cos (z-\alpha \Delta \theta)\right) \text {. }
$$

The contours are as in Fig. 1 b, except that $\theta$ is replaced by $\Delta \theta$.

Just as in the calculation of the wave function, one may separate the contour into a sum of vertical lines plus a closed Cauchy contour around the real poles of $\operatorname{ctn} \frac{z}{2 \alpha}$. We find

$$
\begin{gathered}
G=G_{1}+G_{2}, \\
G_{1}\left(\mathbf{r}, \mathbf{r}^{\prime} ;-i T\right)=\frac{\alpha}{2 \pi \tau} \sum_{n}^{\prime} \exp -\frac{1}{2 \tau}\left(r^{2}+r^{\prime 2}-2 r r^{\prime} \cos \alpha \Delta \theta_{n}\right),
\end{gathered}
$$

where $\Delta \theta_{n}=\Delta \theta-2 \pi n$ and prime insures that this argument of the cosine is between $-\pi$ and $\pi$,

$$
\begin{aligned}
G_{2}\left(\mathbf{r}, \mathbf{r}^{\prime} ;-i T\right)= & \frac{1}{4 \pi} \int_{-\infty}^{\infty} d y\left(\operatorname{ctn}\left(\frac{i y}{2 \alpha}-\frac{\pi}{2 \alpha}-\frac{\Delta \theta}{2}\right)-\operatorname{ctn}\left(\frac{i y}{2 \alpha}+\frac{\pi}{2 \alpha}-\frac{\Delta \theta}{2}\right)\right) \\
& \times \exp -\frac{1}{2 \tau}\left(r^{2}+r^{\prime 2}+2 r r^{\prime} \cosh y\right) .
\end{aligned}
$$

Note that $G_{1}$ and $G_{2}$ are separately discontinuous. [The differences between the propagator and wave function expressions (ctn vs. tan, etc.) may be traced to the fact that $\left(\theta-\theta^{\prime}-\pi\right)$ is really the angle in $G$ corresponding to $\theta$ in $\psi$, since $\Delta \theta-\pi$ corresponds to the forward direction.]

For integer $1 / \alpha, G_{2}$ vanishes and we are left with the finite sum (A.6b). For rational $\alpha=\frac{p}{q}$ one may construct an alternate solution to $(4.15)$, that is the continuous version of $G_{1}$,

$$
G_{0}\left(\mathrm{r}, \mathbf{r}^{\prime} ;-i T\right)=\frac{\alpha}{2 \pi \tau} \sum_{n=0}^{q-1} \exp -\frac{1}{2 \tau}\left(r^{2}+r^{\prime 2}-2 r r^{\prime} \cos \alpha \Delta \theta_{n}\right)
$$


This is obviously the analog to our $\psi_{0}$ wave function.

Both $G$ and $G_{0}$ satisfy the Schrödinger equation on the cone, but obey different initial conditions when time [or $\tau]$ vanishes. From (A.6) and (A.7) it is clear that as $\tau \rightarrow 0, G$ and $G_{0}$ fail to vanish only at the zeroes of $\left(r^{2}+r^{\prime 2}-2 r r^{\prime} \cos \alpha \Delta \theta_{n}\right)$, i.e. when $r=r^{\prime}$ and $\cos \alpha \Delta \theta_{n}=1$. Given the prime limitation on the sum in (A.6b), the only solution for $G$ is $\alpha \Delta \theta=0$, and we obtain the usual $\delta\left(\mathbf{r}-\mathbf{r}^{\prime}\right)$ singularity at $\tau=0$. On the other hand, $G_{0}$ in (A.7) has no such limitation on the angular region and $\alpha \Delta \theta_{n}$ $=2 m \pi$ is permitted. This occurs for $\Delta \theta=0,2 \pi\left(\frac{q}{p} \bmod 1\right), \ldots, 2 \pi\left(\frac{p-1}{p} q \bmod 1\right)$. Thus, in general, a set of two-dimensional delta functions is present at initial time, and $G_{0}$, in spite of its greater simplicity, is not a propagator in the usual sense, possessing as it does more than one "source."

Acknowledgement. We thank G. 't Hooft for a discussion of his work, and for providing us with his paper prior to publication.

Note Added. We have learned from R. Seeley of an extensive mathematical literature [7] dealing with diffusion and wave equations on the cone. While we have not found an analysis of precisely our problem, there exist similar results for the heat kernel $[8,9]$. The interested reader should consult the paper by Cheeger [9] where he will also find reference to earlier literature, which extends to a $19^{\text {th }}$-century work by Sommerfeld.

\section{References}

1. Deser, S., Jackiw, R., ‘t Hooft, G.: Ann. Phys. 152, 220 (1984)

2. Deser, S., Jackiw, R.: Ann. Phys. 153, 405 (1984)

3. For a review see, Jackiw, R.: Nucl. Phys. B 252, 343 (1985)

4. Staruszkiewicz, A.: Acta Phys. Polon. 24, 734 (1963)

Levin, J.: Ph. D. thesis, Brandeis University (1964)

Gott, J., Alpert, M.: Gen. Rel. Grav. 16, 243 (1984)

Giddings, S., Abbott, J., Kuchar, K.: Gen. Rel. Grav. 16, 751 (1984)

5. Marder, L.: In: Recent developments in general relativity. Warsaw: PWN and New York: Pergamon 1962; Proc. Roy. Soc. Lond. Ser. A 244, 524 (1958) and A 252, 45 (1959)

Vilenkin, A.: Phys. Rev. D 23, 852 (1981)

Gott, J.: Ap. J. 288, 422 (1985)

6. 't Hooft, G.: Utrecht preprint THU-88-5. Commun. Math. Phys. 117, 685-700 (1988)

7. Bruening, J., Seeley, R.: J. Funct. Anal. 73, 369 (1987)

8. Dowker, J.: J. Phys. A 10, 115 (1977)

Linet, B.: Ann. Inst. Henri Poincaré 45, 249 (1986)

DeWitt-Morette, C., Low, S., Schulman, L., Shiekh, A.: Found. Phys. 16, 311 (1986)

9. Cheeger, J.: J. Differ. Geom. 18, 575 (1983)

Communicated by L. Alvarez-Gaumé

Received February 19, 1988 
